\title{
Design of a HOM Coupler for a Damped Cavity at the Photon Factory Storage Ring
}

\author{
M. Izawa, S. Sakanaka, T. Takahashi", Photon Factory, KEK, Ibaraki, 305-0801, Japan \\ T. Koseki, Y. Kamiya, ISSP, University of Tokyo, Tokyo 188-8501,
}

\begin{abstract}
We designed a higher order mode (HOM) coupler with a rod-type antenna to reduce HOM's impedances of trapped modes in a damped cavity. The antenna is followed by a standard coaxial line. A tapered siliconcarbide $(\mathrm{SiC})$ load is fixed at the end of the coaxial line. The HOM coupler is attached to an opening for the fixed tuner which is used to detune the resonance frequencies of trapped modes to avoid the coupled-bunch instabilities. The shape of the $\mathrm{SiC}$ load and the insulator for the inner conductor of the coaxial line is optimized so that an input SWR is as small as possible by using the computer code HFSS [1]. The input SWR was obtained to be less than 1.7 over the wide frequency range between 0.7 to 1.4 GHz. The low power measurement showed this type of HOM coupler sufficiently damped the HOM's in the cavity. The fabrication of the high power model based on this design is in progress.
\end{abstract}

\section{INTRODUCTION}

We have developed a damped structure RF cavity for a high brilliance configuration of Photon Factory (PF) storage ring [2] and a VSX project of the University of Tokyo [3]. The four damped cavities have been operating in the PF ring since 1997 [4]. The damped cavity has a large diameter beam duct. HOMs whose frequencies are above the cut-off frequency of the beam duct propagate out from the cavity to the beam duct, and the HOMs are damped by the $\mathrm{SiC}$ absorber which is placed inside wall of the beam duct. The several HOMs, with frequencies lower than the cut-off, still remain in the cavity with high $Q$ values and have the possibility of introducing coupledbunch instabilities. These HOMs frequencies are detuned so as not to introduce any coupled bunch instabilities using two fixed tuners of the cavity in the PF storage ring. For a ring with a larger circumference such as the VSX ring, the frequency detuning becomes less effective, because of its low revolution frequency. We have developed HOM coupler [5 7] with a rod antenna to reduce $\mathrm{Q}$ values of the trapped HOMs. We fabricated low-power models of the HOM coupler and measured the RF characteristics of the trapped modes. The results of the measurement showed that the HOM coupler could

*Email: takeshi.takahashi@kek.jp sufficiently reduce the Q-values of six out of nine dangerous HOMs without influence on the Q-value of the accelerating mode [8].

\section{DESIGN CONCEPT OF THE HOM COUPLER}

The dangerous trapped HOMs are TM011, TM020, TM021, TE111, TM110 and TM111 mode. These HOM's frequencies are distributed from 0.7 to $1.4 \mathrm{GHz}$. Figure 1 shows a schematic cross-sectional view of the cavity with the HOM coupler. The rod antenna of the HOM coupler is located in the center of the cylindrical wall of the cavity. Therefore the HOM coupler strongly couples with TM011, TE111, TM021, and TM111 mode, and does not couple with the fundamental mode. The input reflection coefficient of the HOM coupler should be small enough over the wide frequency range between 0.7 to $1.4 \mathrm{GHz}$. We set a design goal to achieve an input VSWR of less than 1.7 (10\% power reflection) in the frequency range.

As for the load, we chose SiC (CERASIC-B, Toshiba Ceramics co. Ltd), which is the same material as that used in the beam duct, since it has the high loss-tangent in the wide frequency range, the high thermal conductivity and the low outgassing rate, and is working well as the HOM absorber of the damped cavity in the PF ring [9 11].

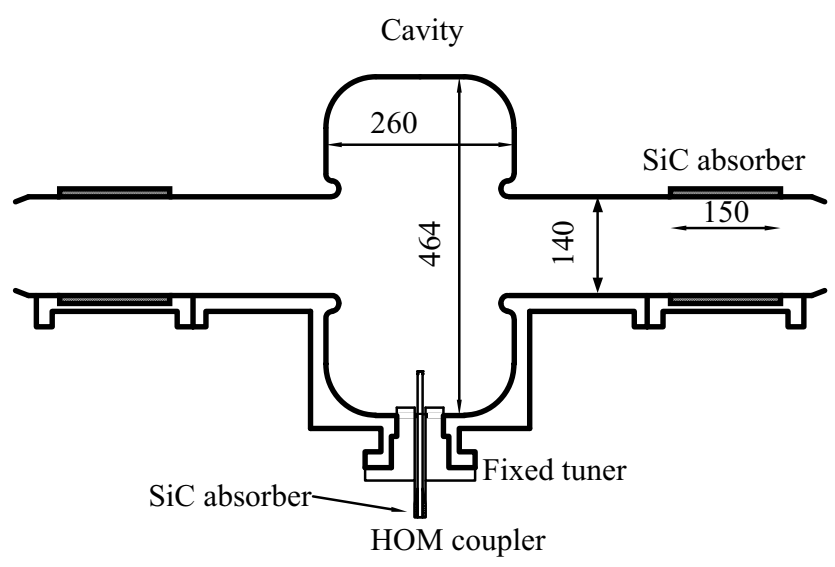

Figure 1. Cross-sectional view of the cavity with the HOM coupler. 


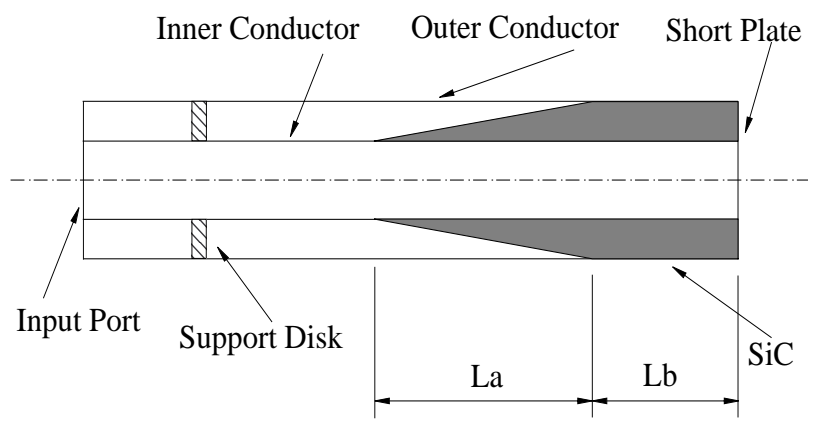

Figure 2. A simulation model of the HOM load.

\section{DESIGN OF THE HOM ABSORBER}

The small size HOM coupler is preferable to avoid the interference with other ring components such as synchrotron radiation beam line. To satisfy this requirement, we designed the structure of the HOM coupler by using the computer simulation codes HFSS. Figure 2 shows an example of the simulation model. We set the basic design of the HOM coupler as follows. The diameters of the inner and outer conductors are $10 \mathrm{~mm}$ and $20 \mathrm{~mm}$, respectively. The inner conductor is supported by a support disk and the $\mathrm{SiC}$ absorber which is fixed between the outer and inner conductors at the end of the coaxial line. The end of the coaxial line is shorted by metal (short plate) to seal vacuum. The SiC absorber is made in the shape of a taper to reduce reflections. We calculated reflection characteristics of the taper of the $\mathrm{SiC}$. Figure 3 shows the reflection characteristics versus frequency on the taper section length (La) of the $\mathrm{SiC}$ without support disk.

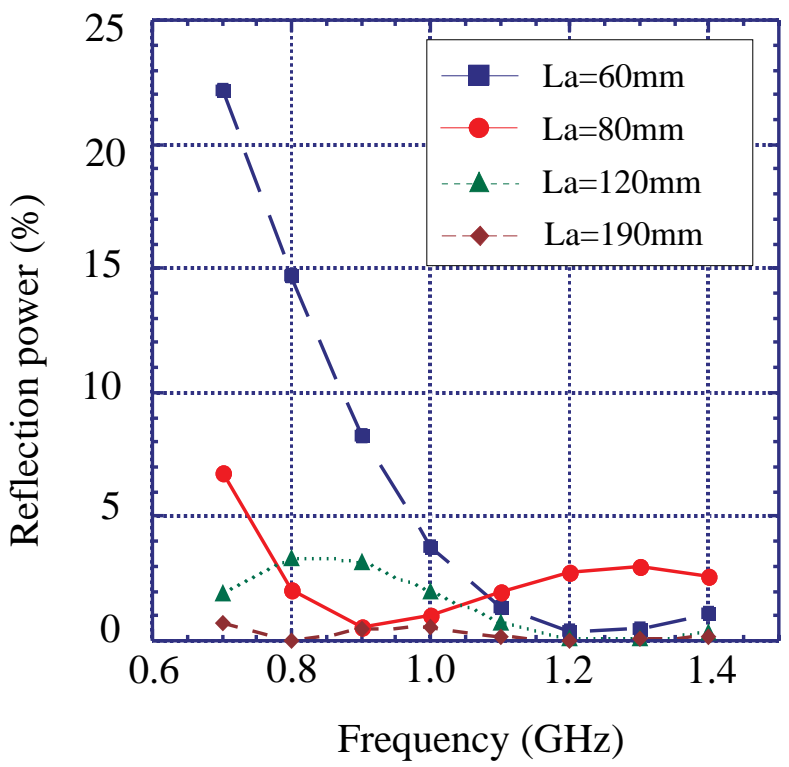

Figure 3. Reflection characteristics of $\mathrm{SiC}$ absorber on some taper lengths $\mathrm{La}=60,80,120$ and $190 \mathrm{~mm}$.
The calculation conditions are as follows. Straight section length $(\mathrm{Lb})$ of $\mathrm{SiC}$ absorber is $80 \mathrm{~mm}$ that is long enough to absorb incident power completely. The reflections from the short plate can be ignored. We assumed that the dielectric constants of the $\mathrm{SiC}$ are a permittivity $\varepsilon^{\prime}=25$ and $\tan \delta=1.4$, which are the approximate values of the beam duct SiC. The longer the taper length (La), the lower reflection coefficient is obtained as expected. As seen in Fig. 3, the reflection power of the taper section of the $\mathrm{SiC}$ become lower than $10 \%$ at a taper length La of $80 \mathrm{~mm}$. Then we adopted the taper length La of $80 \mathrm{~mm}$, and cut the thin part (below $2 \mathrm{~mm}$ in thickness) of the taper, since a thin $\mathrm{SiC}$ is fragile. Figure 4 shows our final design. The taper length (La) is $48 \mathrm{~mm}$ and the minimum thickness of the $\mathrm{SiC}$ absorber is $2 \mathrm{~mm}$. The straight section length $(\mathrm{Lb}=10 \mathrm{~mm})$ of the $\mathrm{SiC}$ absorber is not long enough to completely absorb incident power. However, the straight section length ( $\mathrm{Lb}$ ) and the distance between the support disk and the $\mathrm{SiC}$ absorber were adjusted, so that the reflection waves from the support disk, the SiC absorber, and the short plate (the end of the $\mathrm{SiC}$ absorber), cancel each other. The $\mathrm{SiC}$ absorber is divided into four pieces since they are easily brazed to the inner conductor. The inner and outer conductors have cooling water channels. The $\mathrm{SiC}$ is cooled via the inner conductor. The support disk is located at $80 \mathrm{~mm}$ distant from the cavity wall, where an electromagnetic field strength of the fundamental mode is weak enough.

The influence of the support disk on the reflection characteristics is very important in our design. Figure 5 shows the frequency dependence of the reflection power on three different types of support disk materials, alumina ceramics (permittivity $\varepsilon^{\prime}=10$ ), macor $\left(\varepsilon^{\prime}=6\right)$ and teflon $\left(\varepsilon^{\prime}=2\right)$. As seen in Fig. 5, the reflection power is lower than $7 \%$ in the frequency range $0.7-1.4 \mathrm{GHz}$ for three support disk materials.

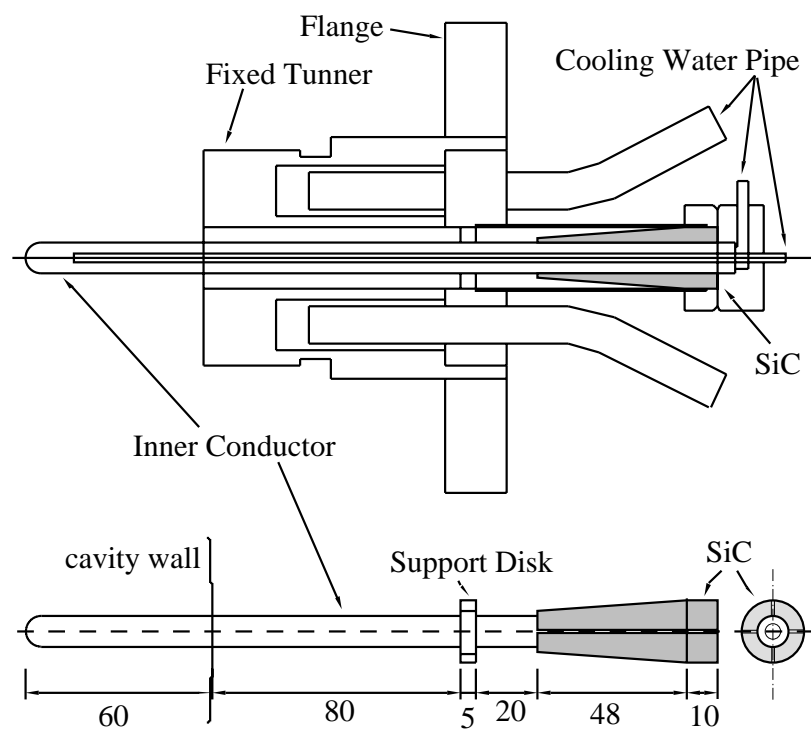

DITAILS OF INNER CONDUCTOR AND SIC ABSORBER

Figure 4. The final design of the HOM absorber. 


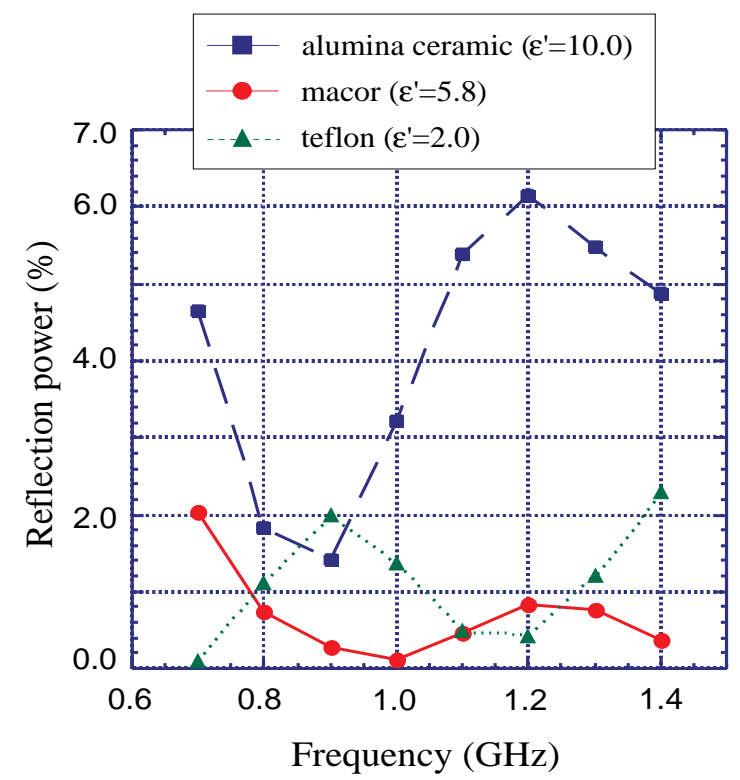

Figure 5. Reflection characteristics of our final design of the HOM coupler, for three different types of the support disk materials alumina ceramics $\left(\varepsilon^{\prime}=10\right)$, macor $\left(\varepsilon^{\prime}=5\right)$ and teflon $\left(\varepsilon^{\prime}=2\right)$.

We adopted the macor as the support disk material since the reflection coefficients become below $2 \%$.

The reflection characteristics are affected also by the dielectric constant of $\mathrm{SiC}$ which changes easily depending on the fabrication condition. Figure 6 shows the effect of the dielectric constants of the $\mathrm{SiC}$ on the reflection characteristics.

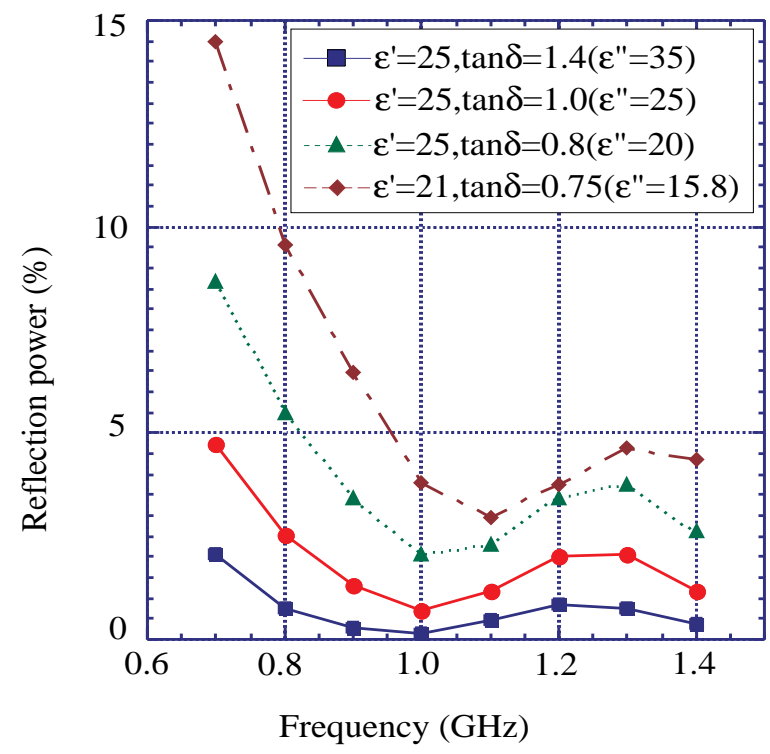

Figure 6. Reflection characteristics of our final design of the HOM coupler, for some different dielectric constants of the $\mathrm{SiC}$.
The required permittivity is about $\mathrm{e} "=\sim 20$ to satisfy our design goal as shown in Fig. 6. We fabricated $\mathrm{SiC}$ samples to verify the process of manufacturing the $\mathrm{SiC}$ absorber and measured the complex dielectric constant. The measured values were $\varepsilon^{\prime}=20 \sim 21, \tan \delta=\sim 1.0\left(\varepsilon^{\prime \prime}=\right.$ $\sim 20)$ at $1 \mathrm{GHz}$. These dielectric constants are barely acceptable to achieve our design goal. The $\mathrm{SiC}$ absorber of our final design is being manufactured in Toshiba corporation.

\section{CONCLUSION}

The HOM coupler with a SiC absorber was designed by the use of the computer code HFSS. The VSWR of the HOM coupler could be less than 1.7 (10\% power reflection) in frequency range $0.7-1.4 \mathrm{GHz}$. The dielectric constants of the $\mathrm{SiC}$ samples were found to be barely acceptable. The HOM coupler will be tested at the PF ring.

\section{ACNOWLEDGMENTS}

We would like to thank Yasunao Takeuchi for valuable discussions and offering useful data of $\mathrm{SiC}$ properties. And we also acknowledge their valuable advises to $\mathrm{K}$. Satoh and other members of Toshiba corporation. We would like to thank Dir. M. Kobayashi for his encouragement.

\section{REFERENCES}

[1] Hewlett-Packard Company.

[2] M. Izawa, T. Koseki, et al., "Installation of new damped cavities at the Photon Factory storage ring", Journal of Synchrotron Radiation (1998). 5, 369-371

[3] H. Takaki, et al., "A Lattice for the Future Project of VUV and Soft X-Ray High-Brilliant Light Source", 1997 PAC, Vancouver, May 12-16 1997

[4] M. Kato, et al., "Reconstruction for the brilliance-upgrading project of the Photon Factory storage ring", Journal of Synchrotron Radiation (1998). 5, 366-368

[5] M. Izawa, et al., "Higher-Order-Mode Damping Coupler for Beam Instability Suppression", Japanese Journal of Applied Physics, Vol. 26, No. 10, October, 1987, pp. 1733-1739

[6] R.Sundelin, M. Billing, R. Kaplan, J. Kirchgessner, R. Meller, D. Morse, L. Phillips, D. Rice, J. Seeman and E. von Borstel: IEEE Trans. Nucl. Sci. NS-28 (1981) 2844

[7] Y. Yamazaki, K. Takata and S. Tokumoto: IEEE Trans. Nucl. Sci. NS-28 (1981) 2915

[8] Y. Kamiya, et al., "HOM Coupler for the Damped Cavity of High Brilliance SR Source", 6th

[9] S. Sakanaka, et al., "Development of a Broadband HOM Load for the 714-MHz HOM-Damped Cavity", 1997 PAC, Vancouver, May 12-16 1997

[10] Y. Takeuchi, et al., "HOM Absorber for the ARES Cavity", 1997 PAC, Vancouver, May 12-16 1997

[11] M. Izawa, et al., "Operation of new rf Damped Cavity at the Photon Factory storage ring”, APAC98, KEK, Tsukuba, Japan, , March 23-27 1998 